\title{
New contributions into the Dezert-Smarandache theory: Application to remote sensing image classification
}

\author{
Fatma Haouas \\ Department of Computer Science, FSM \\ Laboratory: LIPAH \\ Monastir, Tunisia \\ Email: fatmahaouas@hotmail.com
}

\author{
Zouhour Ben Dhiaf \\ Department of Computer Science, FST \\ Laboratory: LIPAH \\ Tunis, Tunisia \\ Email: zouhour_bendhiaf@yahoo.fr
}

\begin{abstract}
The theory of Dezert and Smarandache (DSmT) called also the theory of plausible and paradoxical reasoning represents a suitable formalism to fuse paradoxical sources. The application of the DSmT needs at first the generation of the hyper-powerset and more exactly the composites events. Thus, we present in this paper a new approach to construct pertinent paradoxical classes based on gray levels histograms, which allows also the reduction of the cardinality of the hyper-powerset. Then, we developed a new technique for ordering and coding generalized focal elements. This technique is exploited, in particular, to calculate the cardinality of Dezert and Smarandache. Finally, we applied this new methods to process the classification of a hight resolution images of a forest area.
\end{abstract}

Keywords-Fusion, Dezert-Smarandache theory, Paradoxical class, Coding and ordering, DSm Cardinality

\section{INTRODUCTION}

The Data fusion represents an important approach of information processing which can be applied in many areas to resolve complex problems. Wald defined the fusion process as a formal framework in which are expressed the means and tools for the alliance of data originating from different sources [1]. So, the fusion aims to exploit available information, redundancy and complementarity to arrive at a better reading and understanding of the phenomenon treaty, through a number of steps and using a variety of techniques.

In general, the fusion aims to reduce information imperfections such as uncertainty, imprecision and conflict which represent the most difficult imperfections of information to handle, and then leads to perfect and certain information. The multi-sources fusion becomes one of the important techniques of information processing in several areas in which the information to be merged, objectives and methods may vary remarkably [2], [3]. Thus, the fusion can be applied to process the classification and change detection of remote sensing image, which have spatial, spectral and temporal different characteristics.

The Dempster-Shafer theory is one of the most used theory to fuse information and performs classification [4]. But this later fails in front of highly conflicting sources [5].
Yet the new theory of Dezert and Smarandache can deals with this problem.

This paper presents some contributions into the recent theory of plausible and paradoxical reasoning (DSmT) in order to apply it in data fusion. The modeling in the DSmT requires the generation of the hyper-powerset which hold in the singletons, uncertain classes and paradoxical classes. Thus, we established a new method to determine the paradoxical classes taking advantage the spectral information. Then, to avoid ambiguity between compound classes and facilitate the task of combining, a new technique for coding and ordering classes was developed. We propose a new method to calculate the DSm cardinallity by exploiting the later technique. Finally, we applied the new contributions into the DSmT and the DST to perform the classification of a very high resolution images of a forest area.

\section{Foundations OF THE DEMPSTER SHAFER THEORY}

The Dempster-Shafer theory (DST) called also the belief functions theory was concieved by Dempster and developped by Shafer [4], [6], [7]. It is based on the mass function $m($. and allows the process of imperfect data such as uncertainty, imprecision, ignorance, ambiguity and low conflict.

One advantage of the DST is its rich modeling since it reasons on the set of all the disjunction of hypothesis $2^{\Omega}$ which is called the powerset. Where $\Omega$ is the frame of discernment which contains the hypothesis that model the problem. It must be exhaustive and exclusive.

The mass function $m($.$) represents the basic function of$ the DST it associates to the sub-set of the $2^{\Omega}$ a confidence degree in $[0,1]$ :

$$
\begin{cases}m(\Omega) & =1 \\ \sum_{A \in 2^{\Omega}} m(A) & =1\end{cases}
$$

The masses corresponding to the simple assumptions express the certainty of an hypothesis relative to the others, whereas the masses corresponding to composed assumptions express the confusion associated to the lack of information to decide between an hypothesis or other. Otherwise, the 
masses of compound entities represent ignorance. The elements of powerset that have nonzero masses are called focal elements.

The belief and plausibility functions derived from the mass function defined form $2^{\Omega}$ in $[0,1]$ :

$$
\forall A \in 2^{\Omega}, \quad\left\{\begin{array}{l}
\operatorname{Bel}(A)=\sum_{B \subseteq A} m(B) \\
P l(A)=\sum_{B \cap A \neq \emptyset} m(B)
\end{array}\right.
$$

Where $\operatorname{Bel}(A)$ represents the confidence in $A$ and $\operatorname{Pl}(A)$ denotes the plausibility that the truth is in $A$.

In general, the famous orthogonal sum rule of Dempster is used to combine the masses of evidence. For tow sources $S_{1}$ and $S_{2}$ the normalized form is given by the following expression [8]:

$$
\begin{cases}K & =\sum_{C_{1} \cap C_{2}=\emptyset} m^{s_{1}}\left(C_{1}\right) * m^{s_{2}}\left(C_{2}\right) \\ \forall A \in 2^{\Omega}, m(A) & =\sum_{B \cap C=A} \frac{m^{s_{1}}(B) * m^{s_{2}}(C)}{1-K}\end{cases}
$$

With $m^{s_{1}}$ and $m^{s_{2}}$ represent the masses function of the $S_{1}$ and $S_{2} . K$ is called the mass of the empty or inconsistency of fusion. Despite this normalized form can combine conflicting sources, but it is unable to combine highly conflicting masses distributions [9].

Following the combination phase, the last stage of decision is usually done by the maximizing of credibility, plausibility or pignistic probability. This latter was introduced by Smets [10].

\section{Foundations of THE DEZERT-SMARANDACHE THEORY}

It represents a new extension of the Demspter-Shafer theory which was developed by Dezert and Smarandache in 2001 [11]-[14]. This recent theory characterized by its rich modeling and its flexibility that allow it solving some problems in which the DST has failed.

The first fundamental difference between the DSmT and DST is the no-exclusivity of the frame of discernment $\Omega$ which is called in this case the generalized frame of discernment. It is modeled by the intersection of the hypotheses that form $\Omega$. From the later the hyper-powerset $D^{\Omega}$ is build using the conjunctiva " $\cap$ " and disjunctive " $\cup$ " operators. Let $\Omega=\left\{C_{1}, C_{2}\right\}$ the hyper-powerset $D^{\Omega}=$ $\left\{\emptyset, C_{1}, C_{2}, C_{1} \cup C_{2}, C_{1} \cap C_{2}\right\}$.

The DSmT generates an exponential complexity much higher then the DST, for example if $|\Omega|=4$ so $\left|2^{\Omega}\right|=16$ and $\left|D^{\Omega}\right|=167$.

The generalized mass function $m($.$) is defined from the$ hyeper-powerset $D^{\Omega}$ in $[0,1]$ :

$$
\begin{cases}m(\emptyset) & =0 \\ \sum_{A \in D^{\Omega}} m(A) & =1\end{cases}
$$

The belief and plusibility functions are defined almost in the same manner as in the DST.
Dezert and Smarandache proposed a variety of combination rules able to merge conflicting masses distributions such as: PCR5 (Proportional Conflict Redistribution version five), DSmH, MURR, URR and PURR [14]-[16].

The generalized form of the orthogonal sum rule is called Dezert-Smarandache classic rule (DSmC). It is given by the following expression:

$$
\forall A \in D^{\Omega}, m(A)=\sum_{B \cap C=A} m^{S_{1}}(B) * m^{S_{2}}(C)
$$

The DSmT offers three models of fusion, which are free model $M^{F}$, hybrid $M$ and Shafer $M^{0}$ [14]. The Free model is obtained when the hyper-powerset considered entirely while the hybrid model results when constraints of exclusivity and non-existence are imposed. We get Shafer's model if all exclusivity constraints are imposed and in this case it is the DST context.

To applied the maximum of pignistic probability within the DSmT Dezert and Smarandache proposed a generalized form which is given by the following expression [17]:

$$
G P T\left(A_{i}\right)=\sum_{A_{j} \in D^{\Omega}} \frac{C_{M}\left(A_{i} \cap A_{j}\right)}{C_{M}\left(A_{j}\right)} * m\left(A_{j}\right)
$$

$C_{M}$ (.) represents the cardinality of Dezert-Smarandache.

Definition: the DSm cardinality of any element $A$ in the hyper-powerset $D^{\Omega}$ is the number of parts of $A$ in the Ven diagram of the problem $M$.

\section{Proposed METHOd FOR GENERATING PARADOXICAL CLASSES}

The first difficulty of a fusion process concerns its first step which is the modeling. Within the DSmT the number of the composed classes can be enormous. Moreover, the composite classes have a specific mining that we must taken it into account for their generation. Indeed, classes composed by the union operator translates the uncertainty and the ignorance, while the classes formed by the conjunction of singletons represent the paradox and the ambiguity. Thus, it appears necessary to find a method to determine them respecting their meanings. Let $D^{\Omega}=\left\{\emptyset, C_{1}, C_{2}, C_{1} \cup\right.$ $\left.C_{2}, C_{1} \cap C_{2}\right\}, m\left(C_{1} \cup C_{2}\right)$ indicates the uncertainty and the absence of information to decide between $C_{1}$ and $C_{2}$ but $m\left(C_{1} \cap C_{2}\right)$ is the paradoxical mass between two singletons and represents ambiguity and inconsistence between them.

To resolve this problem, we used first an approach proposed by Ben Dhiaf in [2] to build uncertain focal elements (classes composed by $\cup$ ). It's based on gray levels histograms of classes. Thus, the histogram of an uncertain class $A$ is formed by the lower bounds of histograms of classes included in class $A$.

Then, we have generalized this approach in order to generate the paradoxical classes (classes composed by the conjunction of the hypotheses). Indeed, we propose to better exploiting and reading the areas of overlap of gray level 
histograms, since they don't represent only the uncertainty but also the ambiguity and conflict.

To build the histogram of a paradoxical class $B$, we consider the maximum frequency of the overlapping area of histograms $h_{C_{i}}$ of classes $C_{i}$ which form by their intersections the class $B$, where $h_{C i}$ represents the histogram of the class $C_{i}$. Thus, the histogram of the class $B$ is given by the following expression:

$$
h_{B}(x)=\max \left\{h_{C h}(x) \mid C_{i} \cap B=B, \quad i \in\{1 . . K\}\right\}
$$

Note that $h_{C h}$ is the histogram function of the area of overlap of the classes that compose by their intersection the class $B$.

Example: Given a generalized frame of discernment formed by three classes non-mutually exclusive $\Omega=\left\{C_{1}, C_{2}, C_{3}\right\}$ such as $C_{1} \cap C_{3}=0$. Their gray level histograms are illustrated by the following figure:

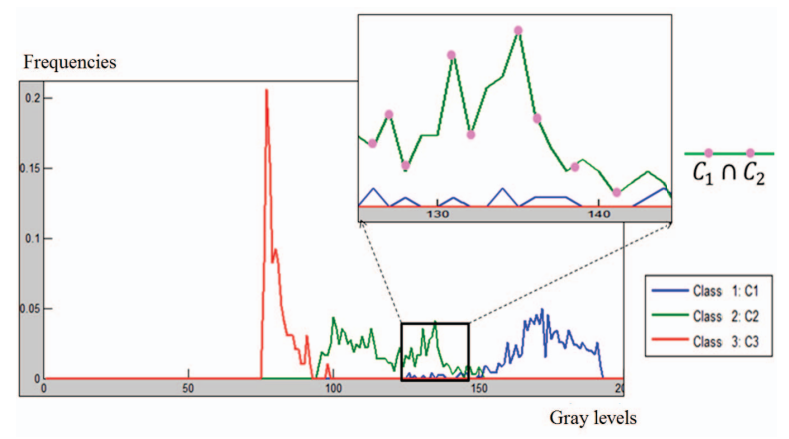

Figure 1: Generation of paradoxical classes.

Considering an overlapping area of $C_{1}$ and $C_{2}$, the histogram of the class $C_{1} \cap C_{2}$ is formed by the highest frequency of the considered area. But these frequencies of the histogram correspond to $C_{2}$. So instead of estimating the mass function on a set consisting of 19 elements we are succeeded to estimate it solely on 7 relevant focal elements.

In this way we have succeeded in express the paradox between classes. We are also managed to determine the generalized focal elements and we have reduced the cardinality of $D^{\Omega}$. This is done by integrating the constraints of exclusivity based on histograms and eliminate insignificant composed classes or those of null masses.

Therefore, we applied the hybrid DSm model and not the free one.

\section{NEW TECHNIQUE FOR CODING AND ORDERING GENERALIZED FOCAL ELEMENTS}

The hyper-powerset typically contains a large number of composed classes. One of the problems that may be encountered during the implementation of the DSmT is the distinction between classes composed by the union and the intersection of singletons (example: $C_{1} \cup C_{2}$ et $C_{1} \cap C_{2}$ ) which generates an ambiguity in the combination phase.
To solve this problem, we propose to build a binary matrix that contains the codes of generalized focal elements and indicating the classes which constitute them and operators that form them. The number of its lines is therefore the number of focal elements. Its columns correspond to the $K$ singletons classes $(K=|\Omega|)$, simple union classes, simple intersection ones and two more others columns to indicate the types of operators used. So the lines contain the codes for focal elements and the columns represent the classes used to build these elements. Thus, the presence of 1 in a given column of a given line indicates either the use of the class of the column whether is the operator of the column. This matrix is ordered such that the first $K$ lines correspond to singletons. The following lines contain the simple union classes followed by simple intersection classes and finally mixed classes i.e those composed by the two operators.

Example: Considering a generalized frame of discernment that satisfying the hybrid model and formed by three classes $\Omega=\left\{C_{1}, C_{2}, C_{3}\right\}$. Given that $C_{2} \cap C_{3}=\emptyset$ then $D^{\Omega}=$ $\left\{C_{1}, C_{2}, C_{3}, C_{1} \cup C_{2}, C_{1} \cup C_{3}, C_{1} \cap C_{2}, C_{1} \cap C_{3}\right\}$. The elements of $D^{\Omega}$ are denoted respectively from $A_{1}$ to $A_{7}$ and corresponding matrix of coding and ordering is given by the following table:

\begin{tabular}{|c|c|c|c|c|c|c|c|c|c|}
\hline & $A_{1}$ & $A_{2}$ & $A_{3}$ & $A_{4}$ & $A_{5}$ & $A_{6}$ & $A_{7}$ & $\cup$ & $\cap$ \\
\hline$A_{1}$ & 1 & 0 & 0 & 0 & 0 & 0 & 0 & 0 & 0 \\
\hline$A_{2}$ & 0 & 1 & 0 & 0 & 0 & 0 & 0 & 0 & 0 \\
\hline$A_{3}$ & 0 & 0 & 1 & 0 & 0 & 0 & 0 & 0 & 0 \\
\hline$A_{4}$ & 1 & 1 & 0 & 0 & 0 & 0 & 0 & 1 & 0 \\
\hline$A_{5}$ & 1 & 0 & 1 & 0 & 0 & 0 & 0 & 1 & 0 \\
\hline$A_{6}$ & 1 & 1 & 0 & 0 & 0 & 0 & 0 & 0 & 1 \\
\hline$A_{7}$ & 1 & 0 & 1 & 0 & 0 & 0 & 0 & 0 & 1 \\
\hline
\end{tabular}

Table I: Example of a matrix for codification elements of $D^{\Omega}$.

\section{Vi. Calculation of the Dezert-Smarandache CARDINALITY}

As we have seen earlier, Dezert and Smarandache proposed a new notion of the cardinality to calculate the generalized pignistic probability [17]. So, we must use the Venn diagram that represents the selected model (free, hybrid or Shafer) and the tackled problem. But there is not a general mathematical rule that allows the calculation of this cardinality for any model of fusion (free, hybrid ...) and any type of classes ( singleton, paradoxical...). Therefore, it creates a difficulty for the generalized pignistic probability calculation above all when the number of focal elements is huge.

To avoid manual calculation of the DSm cardinality of classes, we used the technique of coding to propose a new method that allows us to compute the DSm cardinality of classes. Let $K$ be the number of singleton classes $(K=$ $|\Omega|)$ and $K_{\text {nouv }}$ is the number of singletons classes plus the number of union classes. To calculate the cardinality of 
singleton classes, simple union and simple intersection, we proceed as follows:

- Calculate the cardinality of a singleton class $C_{i}$ :

1) Consider $K_{\text {nouv }}$ first lines of the column $C_{i}$ of the matrix for codification of the problem.

2) $C_{M}\left(C_{i}\right)=$ number of ones in the considered part.

- Calculate the cardinality of simple union class $A$ :

1) Consider the sub-matrix formed by the first $K_{\text {nouv }}$ lines of the matrix for codification.

2) The columns of the sub-matrix corresponding to the classes included in $A$.

3) $C_{M}(A)=$ number of lines that contain at least one 1 .

- Calculate the cardinality of simple intersection class $B$ :

1) Consider the sub-matrix formed by lines of code of simple intersection classes.

2) The columns correspond to classes that form $B$.

3) $C_{M}(B)=$ number of lines that contain only ones.

Example: The Venn diagram corresponding to the example of the paragraph $(\mathrm{V})$ is given by the following figure:

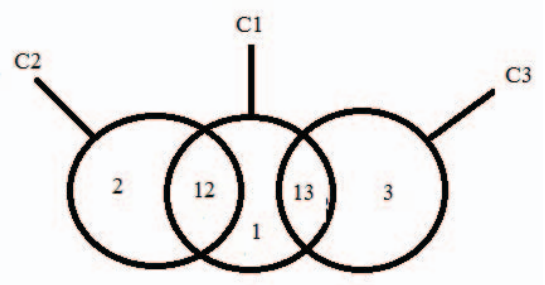

Figure 2: Venn diagram.

For example, to calculate the cardinality of $C_{1}$ which is equal to 3 , we consider the first 5 lines of the column $C_{1}$ in the matrix of the codification $\left(5=K_{\text {nouv }}\right)$.

Thus the cardinality of $C_{1}$ is equal to the number of 1 in the following column which is 3 .

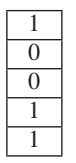

Table II: The cardinality of $C_{1}$.

To calculate the cardinality of $C_{1} \cup C_{3}$, just count the lines that contain at least one 1 in the following sub-matrix:

\begin{tabular}{|l|l|}
\hline 1 & 0 \\
\hline 0 & 0 \\
\hline 0 & 1 \\
\hline 1 & 0 \\
\hline 1 & 1 \\
\hline
\end{tabular}

Table III: The cardinality of $C_{1} \cup C_{3}$.
So, $C_{M}\left(C_{1} \cup C_{3}\right)=4$.

The cardinality $C_{M}\left(C_{1} \cap C_{2}\right)=1$. It is equal to the number of lines that contain only 1 in the following submatrix:

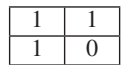

Table IV: The cardinality of $C_{1} \cap C_{2}$.

\section{APPLICATION}

The considered site is a forest area. This zone is characterized by the diversity of stratum and abundance of forest species. In order to realize a supervised classification of a very hight resolution images taken by the Quickbird satellite in 2006, we applied two process of classification by the DST and the DSmT in a punctual framework (pixel level) taking into account the spectral information.

First, we perform the classification of the first hight resolution image (HRI1) by fusing it with the corresponding forest inventory (FI1) which was realized in 2000. Then we applied the sames process of classification by the DST and the DSmT to achieve the classification of the HRI2.

The FI represents a set of stratum. Each one is defined as a representative fraction of a population. The FI gives some information about the area such as the density of the populating and volume and increase of wood. The fusion of the HRI with the inventory improves classification mono source by reducing imperfections and allows detecting changes in the forest cover. The high resolution images (HRI) and the forest inventory (FI) are complementary in the two experiences and do not exhibit the same number of classes (the frames of discernment are different). The HRI is formed by four classes that are CZ, CL, SPC, SN so $\Omega_{H R I}=\{C Z, C L, S P C, S N\}$, while the FI is formed only by two classes which are $\mathrm{CL}+\mathrm{CZ}$ and $\mathrm{SPC}+\mathrm{SN}$, then $\Omega_{F I}=\{C Z+C L, S P C\}$. The CL and $\mathrm{CZ}$ are two different tree spices, SPC represents little ground cover and the class $\mathrm{SN}$ is bare ground.

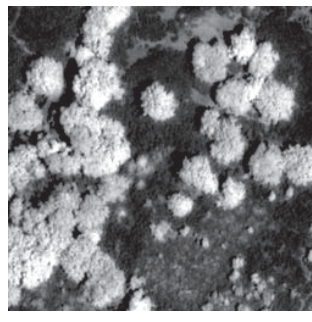

(a) Hight resolution image 1 .

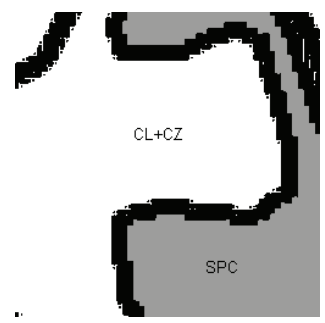

(b) Forest inventory 1
Figure 3: Sources of first experience.

The information provided by the sources is tainted by the imprecision. Indeed, the information acquired by a source does not allow us to decide in some cases the exact 


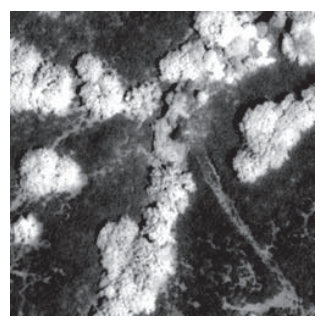

(a) Hight resolution image 2 .

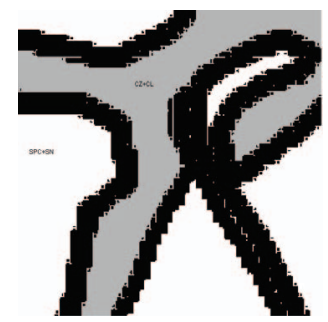

(b) Forest inventory 2.
Figure 4: Sources of the second experience.

belonging of a pixel to a given class. Furthermore, stratum " $C Z+C L$ " represents a mixture of oaks and therefore it symbolizes more than one class. The imprecise nature of the inventory induced uncertainty. For a given pixel image of a HRI, we have available the level of inventory a polygon of the area surrounding it and not a specific information. The

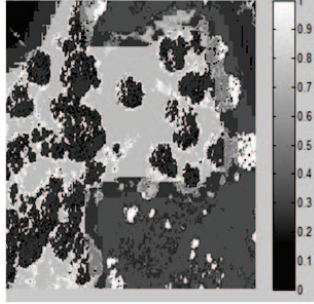

(a)

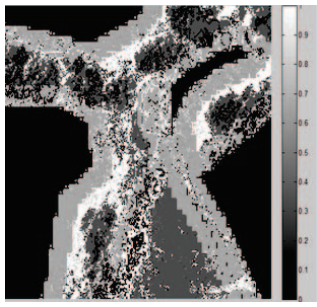

(b)
Figure 5: (a) Image of conflict of HRI1 and FI1,(b) Image of conflict of HRI2 and FI2 .

pixels that have a gray level white or near to white in the images of conflict indicate that the conflict between the two sources is very high. In this case, the conflict is caused first, by the uncertain and imprecise nature of the two sources and second, the time difference of the catches of sources which involves changes that have occurred on the forest cover. To combine the bba within DST and DSmT, we used respectively the DS rule and the its generalized version DSmC. As a first step to estimate the masses functions for the two frameworks (DST and DSmT), we used a probabilist method based on histograms of gray levels defined by Ben Dhiaf [2].

To apply DST, we have weakened the inventory mass by a factor $\alpha=0.8$ to reduce the conflict between the two sources in the two experiences. Visual analysis of the results of classification obtained by the DST show (figures 6,8) that this theory has misclassified conflicting pixels between the two sources. Specially the pixels between the classes $C Z$, $C L$ and $S P C$. They are falsely attributed to $C Z$. Indeed, this pixels represent the change that affected forest cover and the progress of species $\mathrm{CZ}$ and $\mathrm{CL}$ and remember that the sources were not obtained in the same period.

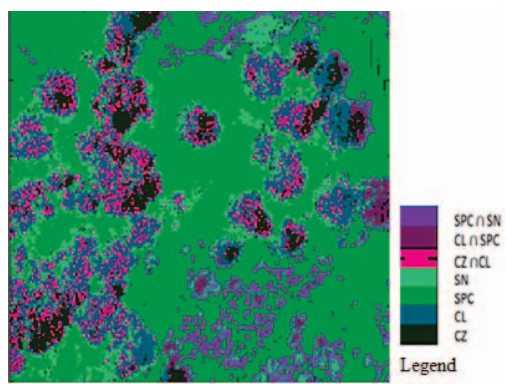

(a) Classification by the DSmT of the HRI1.

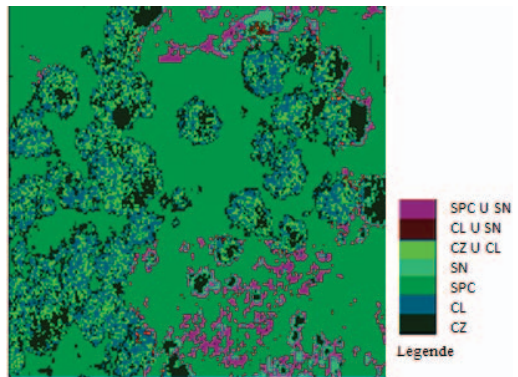

(b) Classification by the DST of the HRI1.

Figure 6: Results of spectral classification of the HRI1.

Whereas, in the first experience the DSmT assigned correctly these pixels to SN class. In addition, conflicting pixels between the classes SPC and CL are putted in a paradoxical class which is $C L \cap S P C$ (fig:7). Similarly, the edge pixels of the CL class with the SN class are assigned to the class $\mathrm{CZ}$ what is wrong. Yet, the DSmT correctly assigned these pixels to $\mathrm{SN}$ class.

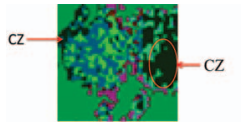

(a)

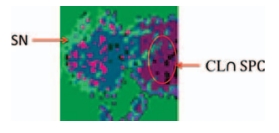

(b)

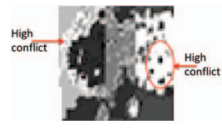

(c)
Figure 7: Comparison of results: (a): DST, (b): DSmT, (c): Conflicting pixels.

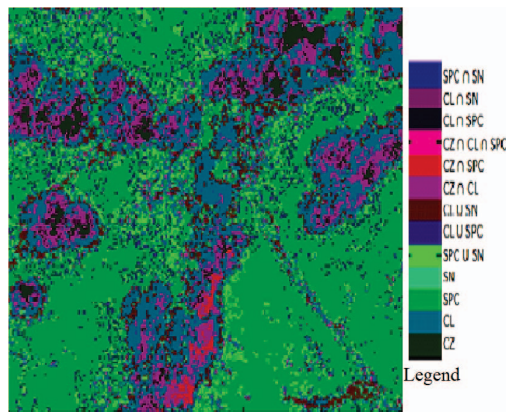

(a) Classification by the DSmT. 


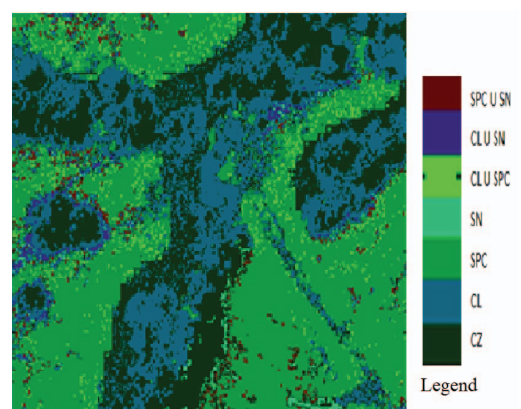

(b) Classification by the DST.

Figure 8: Results of spectral classification of the HRI2.

Moreover, in the second experimentation (fig: 8) the DSmT classed correctly this pixels and attributed them to $S P C$ or $C L$ classes.

In addition, the pixels of the edges between the two spices of tree $C Z$ and $C L$ which represent an ambiguity are classified in a paradoxical class that is $C Z \cap C L$ by the DSmT. This ambiguity was created by the forest inventory since it confuses these two classes.

This results prove the effectiveness of the proposed method to build paradoxical classes and the ability of the DSmT to fuse high contractual sources

\section{CONCLUSION}

In order to achieve the classification of a very high resolution images taken by the QuickBird satellite, we applied two process of classification by the DST and the DSmT.

The process of fusion by the DSmT presents in particular three new contributions.

The first one is a new method that allows generation of paradoxical classes (formed by the intersection operator) based on gray levels histograms. In addition, this later lets imposing constraints of exclusivity and subsequently reduce the number of classes composed therefore the cardinality of the hyper-powerset $D^{\Omega}$. Then, we presented a method based on a simple binary matrix of coding and ordering which was used to give a specific order to the codes of generalized focal elements. This later, allows the distinction between the composed classes specially when they are formed by the same hypotheses but not the same operators. The major advantage of the technique of coding and ordering manifests with the third contribution. Indeed, in order to calculate the cardinality of DSm we used the later technique and we proposed a new method to compute the new cardinality for same classes. The results obtained by the two process prove the ability of the DSmT to manage the conflict between sources better then the DST and the ability of our method to generate correctly the paradoxical classes.

\section{REFERENCES}

[1] L. Wald, "Some terms of reference in data fusion," Geoscience and Remote Sensing, IEEE Transactions on, vol. 37, no. 3, pp. 1190-1193, 1999.

[2] Z. Ben Dhiaf, "Fusion de sources d'information pour l'interprètation d'images de télédétection moyenne et haute résolution : Application l'inventaire forestier," Ph.D. dissertation, Université de Tunis El Manar : Faculté des Sciences Tunis, 2011.

[3] X. Li, X. Huang, and M. Wang, "Robot map building from sonar sensors and dsmt," Information \& Security Journal, Bulg. Acad. of Sci., Sofia, vol. 20, 2006.

[4] G.Shafer, "Perspectives on the theory and practice of belief functions," International Journal of Approximate Reasoning, vol. 4, no. 5, pp. 323-362, 1990.

[5] A. Martin, "Le conflit dans la théorie des fonctions de croyance." in EGC, 2010, pp. 655-666.

[6] G. Shafer, “A mathematical theory of evidence." Princeton University Press, 1976.

[7] P. Vannoorenberghe, "Un état de l'art sur les fonctions de croyance appliquées au traitement de l'information," Revue I3, vol. 3, no. 2, pp. 9-45, 2003.

[8] G. Shafer and R. Logan, "Implementing dempster's rule for hierarchical evidence," Artificial Intellignece,, vol. 33, pp. 271-298, 1987.

[9] A. Tchamova and J. Dezert, "On the behavior of dempster's rule of combination and the foundations of dempster-shafer theory," in Intelligent Systems (IS), 2012 6th IEEE International Conference. IEEE, 2012, pp. 108-113.

[10] P. Smets, "Constructing the pignistic probability function in a context of uncertainty," in Uncertainty in artificial intelligence, vol. 5, 1990, pp. 29-39.

[11] J. Dezert, "Foundations for a new theory of plausible and paradoxical reasoning," Information and Security, vol. 9, pp. 13-57, 2002.

[12] J. Dezert and F. Smarandache, "An introduction to DSmT," arXiv preprint arXiv:0903.0279, 2009.

[13] L. Cholvy, "Non-exclusive hypotheses in dempster-shafer theory," International Journal of Approximate Reasoning, vol. 53, no. 4, pp. 493-501, 2012.

[14] J. Dezert and F. Smarandache, "Dsmt: A new paradigm shift for information fusion," arXiv preprint cs/0610175, 2006.

[15] J. Dezert and F. Smarandache, "Introduction to the fusion of quantitative and qualitative beliefs," Information \& Security Journal, vol. 20, 2006.

[16] J. Dezert and F. Smarandache, "The DSmT approach for information fusion and some open problems," 2005.

[17] J. Dezert, F. Smarandache, and M. Daniel, "The generalized pignistic transformation," arXiv preprint cs/0409007, 2004. 